\title{
Pulse-phase spectroscopy as a tool for the study of X-ray pulsars
}

\author{
Alexander Lutovinov* \\ Space Research Institute, Russia \\ Astronomy Division, Department of Physics, FI-90014 University of Oulu, Finland \\ E-mail: aaleiki.rssi.ru
}

\section{Sergey Tsygankov}

Finnish Centre for Astronomy with ESO (FINCA), University of Turku, Väisäläntie 20, FI-21500

Piikkiö, Finland

Astronomy Division, Department of Physics, FI-90014 University of Oulu, Finland

Space Research Institute, Moscow, Russia

E-mail: tsygankoveiki.rssi.ru

\begin{abstract}
The pulse-phase resolved spectroscopy at different luminosities using for the study of the matter distribution in X-ray pulsars requires a new generation of instruments with high timing and spectroscopic capabilities. Based on the current results of the analysis of the emission from transient X-ray pulsars (e.g., V0332+53) obtained with the RXTE observatory we demonstrated a strong variability of the source spectral parameters with the pulse phase. We also showed that properties of the fluorescent iron line emission (particularly a variability of its equivalent width on the time scale of the pulse period) can be used to investigate the spatial distribution and physical conditions of the matter around the compact object.
\end{abstract}

Fast X-ray timing and spectroscopy at extreme count rates: Science with the HTRS on the International $X$-ray Observatory - HTRS2011,

February 7-11, 2011

Champéry, Switzerland

\footnotetext{
* Speaker.
} 


\section{Introduction}

Geometrical and physical properties of the matter near the neutron star surface could and should be reflected in observational properties of the X-ray pulsars emission. Particularly, strong effects should be seen near the cyclotron energy since the shape of the absorption line depends strongly on the viewing angle (see, e.g., [1], 2]). Parameters of the continuum spectrum should also depend on the viewing angle due to a variable opacity of the accreted matter near the neutron star surface. Thus the pulse phase-resolved spectroscopy can be used as a tool for the study of the properties of the matter around X-ray pulsars at different states and luminosities.

The transient X-ray pulsar V0332+53 is one of the brightest transient X-ray sources on the sky. This fact gives us an unique possibility to perform the pulse phase-resolved spectroscopy of its emission with a high statistical significance using narrow pulse-phase bins. A very powerful outburst from this source was detected in 2004-2005 by the RXTE and INTEGRAL observatories [3, 4]. In the following papers [5, 6] it was shown that the pulse-averaged spectrum of V0332+53 and its parameters are highly variable with the source luminosity. In particular, it was shown that the cyclotron energy is inversely proportional to the source bolometric luminosity and such a dependence is the same at both rising and declining parts of the outburst.

Below we summarize briefly current results of the pulse phase-resolved spectroscopy of V0332+53 performed for different luminosity states. More detailed information can be found in the paper of Lutovinov et al. (in preparation).

\section{Observations and Data Analysis}

This short report continue series of publications [5, 6, 7] devoted to all-round studies of the unique outburst from the transient X-ray pulsar V0332+53 in 2004-2005. In this work we are using the same set of the RXTE observatory data as in previous one [6], which covers both brightening and declining parts of the outburst with different luminosity levels.

To derive the pulse phase-resolved spectra from the PCA and HEXTE instruments we used standard programs of the FTOOLS/LHEASOFT 6.3.2 package and algorithms described in the RXTE cookbook ${ }^{1}$. For observations with high and low intensities photons were folded into 16 and 8-bins pulse profiles, respectively. The obtained pulse-bin spectra were approximated by a powerlaw model with an exponential cutoff (cutoffpl in the XSPEC package), modified by one or two harmonics (main and first) of the cyclotron absorption line in the form

$$
\exp \left(\frac{-\tau_{c y c l}\left(E / E_{c y c l}\right)^{2} \sigma_{c y c l}^{2}}{\left(E-E_{c y c l}\right)^{2}+\sigma_{c y c l}^{2}}\right),
$$

where $E_{c y c l}, \sigma_{c y c l}$ and $\tau_{c y c l}$ are the center, width and depth of the line, respectively [8]. This is the same model which was used earlier for the analysis of pulse-averaged spectra and reasons of its choosing were described in [5, 6]. Note, that the first harmonic was confidently observed and its parameters were restricted not in all data sets, therefore we examined possible effects of its including in the fit on parameters of the main harmonic and the continuum. As it was done for

\footnotetext{
${ }^{1}$ http://heasarc.nasa.gov/docs/xte/recipes/cook_book.html
} 

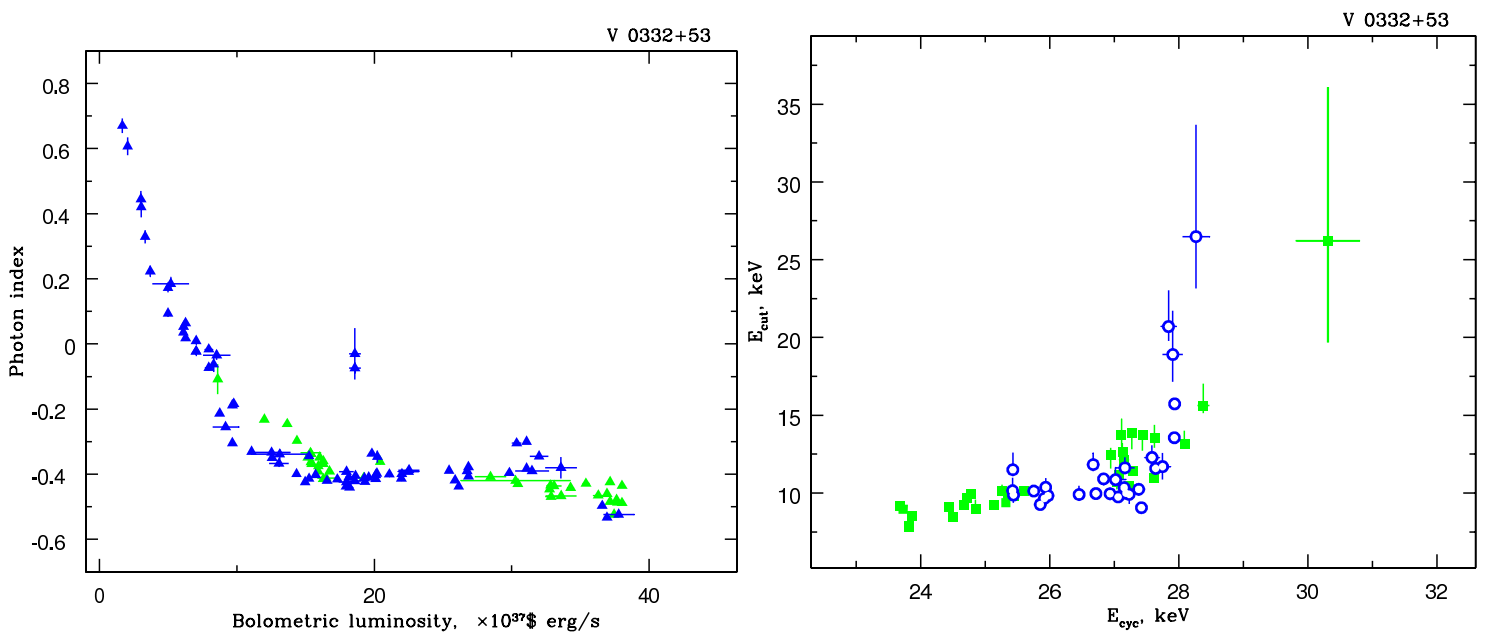

Figure 1: (left) Dependence of the photon index on the V $0332+53$ bolometric luminosity. Green points correspond to the rising part of the outburst, blue ones - to the outburst decay. (right) The cutoff energy $E_{\text {cut }}$ derived from the continuum model versus the cyclotron line energy $E_{\text {cycl }}$. The error bars correspond to $1 \sigma$.

pulse-averaged spectra, the central energy of the first harmonic was either a free parameter or fixed to the doubled main harmonic energy. It was found that parameters of the main harmonic and continuum do not depend within errors on the higher harmonics.

Finally, to take into account the iron fluorescence line at $\sim 6.4 \mathrm{keV}$ a Gaussian line component was included in the model as well.

\section{Results}

\subsection{Pulse-averaged spectra}

Prior to make the pulse phase-resolved spectroscopy we investigated the evolution of the continuum parameters with the source luminosity and outburst phase. The dependence of the photon index on the V0332+53 bolometric luminosity (which was calculated as a luminosity in the 3-100 $\mathrm{keV}$ energy band) is presented in Fig. 1 (left). It is clearly seen a significant hardening of the source spectrum with the luminosity increase, that is probably due to the increasing size of emitted regions (accretion columns) and that the observer begin to see their hotter parts.

An another parameter of the continuum is the cutoff energy $E_{\text {cut }}$, that (in a manner) can be considered as a measure of the electron temperature $T_{\mathrm{e}}$ in the hot continuum-emitting domain. This quantity should evidently be dependent on the specific kinetic energy gained by the accretion flow in the neutron-star gravitational potential and deposited near the neutron-star surface. Therefore, $E_{\text {cut }}$ should be correlated positively with $E_{\text {cycl }}$ and negatively with the bolometric luminosity $L_{\mathrm{X}}$. This fact is illustrated in Fig. [1 (right). Note, that no hysteresis behavior is seen in both plots. 

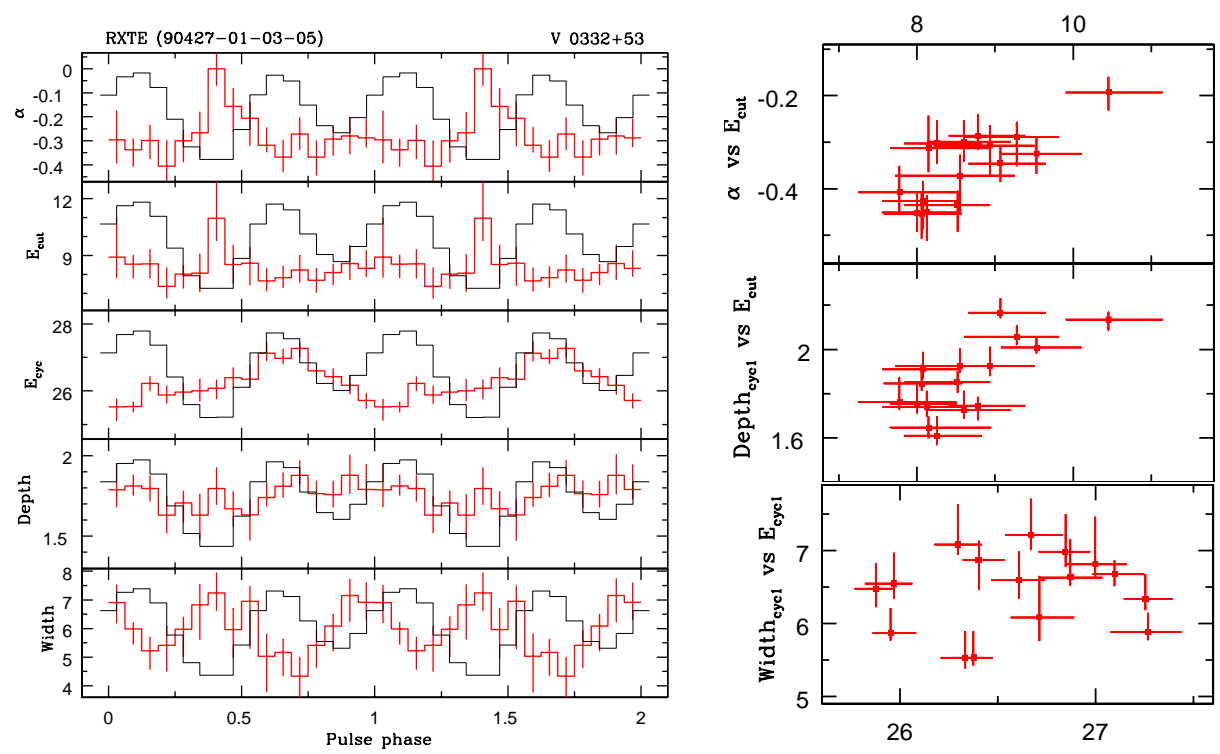

Figure 2: (left) Variability of continuum and cyclotron line parameters (red histograms) with the V0332+53 pulse phase for the source luminosity of $\sim 2 \times 10^{38} \mathrm{erg} / \mathrm{s}$. Corresponding mean pulse profiles in the 20-100 energy band are shown by black histograms. (right) Dependences between different spectral parameters on the time scale of a pulse period for the observation ID 90014-01-03-020, when the source luminosity was about $1.5 \times 10^{38} \mathrm{erg} / \mathrm{s}$. The horizontal axis is expressed in $\mathrm{keV}$.

\subsection{Dependence of the spectral parameters on the pulse phase}

It was mentioned above that different physical conditions of different parts of emission regions and dependence of the emission properties on the viewing angle $\alpha_{\mathrm{obs}}$ should lead to the variability of the spectral parameters with the pulse phase. The typical picture of such a variability is presented in Fig.2 (left) for the observation ID 90427-01-03-05, when the source luminosity was about $2 \times$ $10^{38} \mathrm{erg} / \mathrm{s}$. A comparison with previous results ([9]), obtained for other luminosities, shows that the pulse profile keeps the double-peaked shape in a wide luminosity range, but the maximum of the cyclotron line energy is shifted by $\sim 0.25$ of the pulse period with the luminosity dropping (see Fig.2, left and Fig.3 in [9]). We also built several dependencies between different spectral parameters (Fig. 2, right and found that there is a correlation between the photon index and cutoff energy as well as between the depth of the main harmonic of the cyclotron line and the cutoff energy. In the same time no pronounced linear increase in $\sigma_{\text {cycl }}$ with increasing $E_{\text {cycl }}$ is seen in the pulse phase-resolved spectroscopy, that is agreed with earlier results obtained for pulse-averaged spectra [6].

It is interesting to note, that according to the theory of cyclotron-radiation transfer under neutron-star conditions (see, e.g., [10]), the cyclotron optical depth at the main harmonic varies only slightly with $\alpha_{\mathrm{obs}}$ (see Fig. 2. left) and can hardly exclude the formation of a resonance scattering feature at fairly large $\alpha_{\text {obs. }}$. Hence, the main harmonic non-detection (see, e.g., [11]) should rather be related to specific viewing conditions of the line-forming region such as its small visible area or screening of this region by the neutron star and/or accretion disc/stream.

Finally note, that the pulse phase-resolved spectroscopy gives the unique possibility to trace 


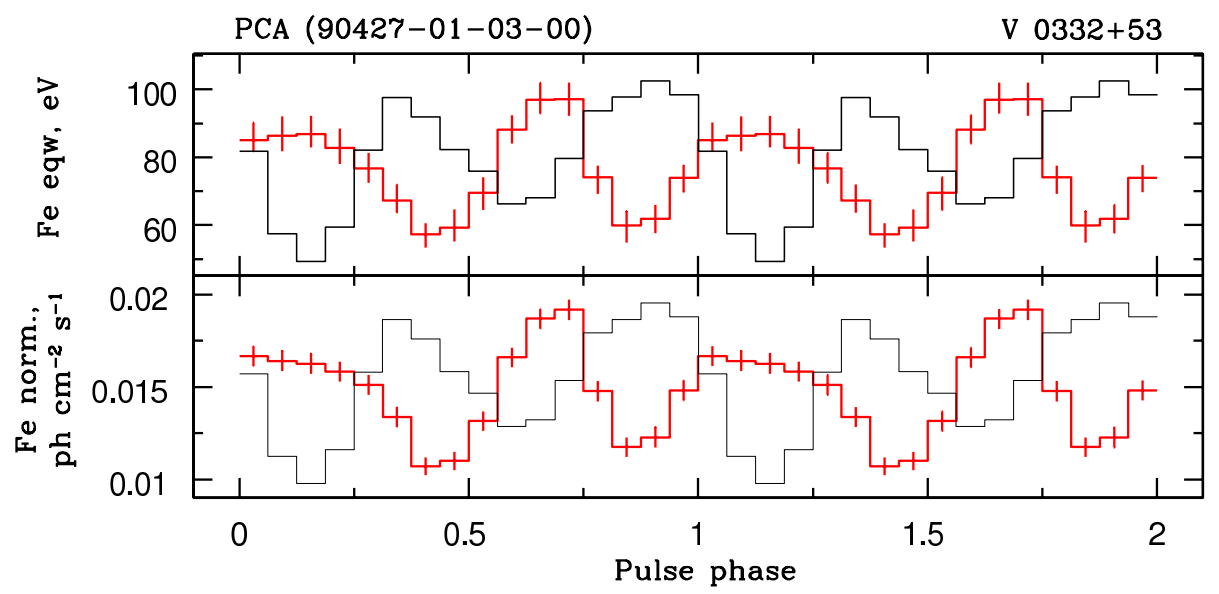

Figure 3: Dependence of the equivalent width and normalization (red histograms) of the iron $K_{\alpha}$ line in the V $0332+53$ spectrum on the pulse phase. The pulse profile (continuum emission) is shown by black histograms.

the evolution of the fluorescent iron $\mathrm{K}_{\alpha}$-line at the time scales much shorter then the pulse period. The goal of such investigations can be a study of a spatial distribution of the matter in the vicinity of the neutron star (汤). The potential of the usage of the continuum photons reverberation was successfully demonstrated in the studies of SMBHs in AGNs (see, e.g., [12]).

The iron line equivalent width and normalization changes are presented in line with ones of the continuum flux over the pulse period during the observation ID 90427-01-03-00, when the source luminosity was $\sim 2 \times 10^{38} \mathrm{erg} \mathrm{s}^{-1}$ (Fig. 3 ). The observable time lag between them $(\sim 1.5 \mathrm{~s})$ can be considered as a time-of-flight of the continuum photons from the source to the reprocessing site (in this case a distance between them $\sim 5 \times 10^{10} \mathrm{~cm}$ is comparable with the outer size of the accretion disk). Another possible explanation of the observed time lag is a phase lag (arising just due to a rotation of the neutron star). In this case the illuminating beam cuts the reprocessing site later than it cuts a direct line of the sight to the observer.

\section{Acknowledgments}

Authors thanks V.Suleimanov and J.Poutanen for useful discussions. This work was partially supported by the Program "The origin, structure, and evolution of objects of the Universe" of the Russian Academy of Sciences, grant NSh-5069.2010.2 for support of leading scientific schools, grant RFBR 11-02-01328 and state contract 14.740.11.0611.

\section{References}

[1] Isenberg M., Lamb D., Wang J., ApJ, 505, (688), 1998

[2] Araya R., Harding A., ApJ, 517, (334), 1999

[3] Swank J., Remillard R., Smith E., ATel, 349, (1), 2004

[4] Tuerler M., Di Cocco G., Diehl R., et al., ATel, 372, (1), 2004 
[5] Tsygankov S., Lutovinov A., Churazov E., Sunyaev R., MNRAS, 371, (19), 2006

[6] Tsygankov S., Lutovinov A., Serber A., MNRAS, 401, (1628), 2010

[7] Tsygankov S., Lutovinov A., PoS, Proceedings of The Extreme sky: Sampling the Universe above 10 keV, 96, (70), 2009 [http://arxiv.org/abs/1002.1898]

[8] Mihara T., Makishima K., Ohashi T., et al., Nature, 346, (250), 1990

[9] Lutovinov A., Tsygankov S., PoS, Proceedings of The Extreme sky: Sampling the Universe above 10 $k e V, 96,(10), 2009$

[10] Zheleznyakov V., Serber A., Astron. Rep., 37, (507), 1993

[11] Staubert R., Chin. J. Astron. Astrophys., 3, (270), 2003

[12] George I., Fabian A., MNRAS, 249, (352), 1991 